\title{
An Investigation into Problems of the English Versions of Northern Shaanxi Folk Songs
}

\author{
Tian Ling \\ School of Foreign Languages, Yan’an University, Yan’an, China \\ lingtian77@126.com
}

Keywords: The English Versions of Northern Shaanxi Folk Songs; Translation; Problems; Interview

\begin{abstract}
Northern Shaanxi folk songs, due to its unique charm, have attracted translators' attention, and there have appeared some English versions. But it's hard to say how the English readers understand and accept them. In order to find out the answer, the author made an interview with the English versions of Lan Hua Hua and Dong Fang Hong as examples. 3 foreigners are interviewed. The result shows that the interviewees can't thoroughly understand the versions. There exist some problems in the translations. To be specific, 4 main problems influence people's understanding, namely, the inaccurate expression, the improper handling of cultural elements, the lack of colloquial language and the unsuitability for singing. Some possible solutions are also proposed.
\end{abstract}

\section{Introduction}

Northern Shaanxi folk songs are well-known for their simple language, rich contents and a strong local flavor. In recent years they are also performed in the Golden Hall of Vienna, so more and more foreigners begin to love those songs. But due to the language barrier, they can't understand the songs. Therefore, attention was aroused among a large number of scholars and they try to translate the songs into English. But there seems to be something unsatisfying in the translated versions. So the paper, on the basis of a general introduction to the translations of Northern Shaanxi folk songs, tries to find out the problems by making an interview to some foreigners and give some suggestions as possible solutions.

\section{A Survey of the Translations of Northern Shaanxi Folk Songs}

Because of the difficulty of song translation and the dialects in Northern Shaanxi folk songs, the number of the English versions is quite limited. Until now two English versions have appeared. One is translated by a professor in Nankai University, Wang Hongyin, while the other is by two middle School Students Han Xiao yue and Wang Xinyu. Voice from the Northwest by Wang Hongyin is a well-classified English version with extensive covering of different kinds. He, according to the topic, classified Northern Shaanxi folk songs into ten parts: The Yellow River, The Great Northwest, Hard Times, Colorful love, How I Miss You, Yangge Dance, Wandering Life, Red Flags Fly, Enter into a New Age and Wandering Chants Forever. Altogether 105 folk songs have been translated. But just as professor Wang Hongyin says, The English versions can be recited, but they can't be sung. They may be called literary translation instead of artistic translation. In my eyes, real artistic translation should be the musical text considering rhythm and rhyme instead of the literary text that mainly focuses on meaning transfer.[1]Soon, two middle school students, Han Xiaoyue and Wang Xinyu, with great enthusiasm in spreading Northern Shaanxi folk songs, the experience of making an interview in the country of Northern Shaanxi, and the advantages of having learned piano and studying in the international class, [2] published a book entitled Translation and Brief Introduction to Some Folk Songs of Northern Shaanxi, which included 50 songs. Surely due to the limit of age and experience, there are some problems and mistakes in this version, but the two girls made a brave attempt, indeed. They have enriched the translations of Northern Shaanxi folk songs. 
What's more, in 2009 the first national academic seminar on the translation of Northern Shaanxi folk songs was held in Xi'an. After it, a collection of the thesis and the translated songs was published, in which Gou Bin translated 27 songs, Wen Shilong 16, Wang Pei, Tian Baolin, Jiao Yueyue and Du Liping 10 respectively, Wang Zhanbin 4 and Dong Qingmin 3. And then in 2012 the second national academic seminar was held in Xi'an, too. In The Collection of the Thesis and the Translated songs, there are 22 by Wen Shilong, 8 by Du Liping, 7 by Ding Ning, 5 by Song Juanjuan and Liu Yujun respectively, 2 by Gou Bin, Jiao Yueyue and Hu Yuanyuan respectively. Wen Shilong and Gou Bin also give new versions for some folk songs translated by them in an earlier time.

The statistics show that more and more scholars begin to be interested in the translation of Northern Shaanxi folk songs and are really doing something for it. Some English versions are suited for singing, while some others only for reading. But according to the present versions it's easy to see that scholars are more interested in some famous and classical folk songs. Therefore, there are different versions for some songs, such as Lan Huahua, Zou Xikou, Nan Niwan, Dong Fanghong, etc. But other less famous songs can't arouse much attention, which leads to the fact that the number of the English versions is limited.

\section{An Investigation into the English Readers' Understanding of the English Versions of Northern Shaanxi Folk Songs}

In order to know how the English readers feel about the English versions of Northern Shaanxi folk songs, we interviewed 3 foreigners. One of them is a female, while the other two are male. They are from America, Britain and Australia respectively, with the age of 28, 35 and 40. One is an overseas student, the others are foreign teachers. The author took the English versions of Lan Hua Hua and Dong Fang Hong as examples and interviewed the 3 foreigners in order to know their opinions about the two English versions and possible factors that obstruct their understanding. The interview was carried out on the basis of two general questions: 1. Can you fully understand the two English versions? Please tell your understanding about them. 2. Is there any sentence that you can't understand? If the answer is yes, how does it influence your understanding? The result shows that the English readers can roughly understand the meaning of the folk songs except for a few sentences, but they can't understand the deep emotions and the cultural connotation. The in-depth communication suggests that the English readers pay little attention to grammatical mistakes since they almost have no influence on their understanding. What leads to the difficulty of understanding is mainly the inaccurate and improper diction. For example, in the English version of Lan Hua Hua, while reading the sentence "She was transported into Zhou's without seeing his true lover", the interviewees can't understand why "transport" is used and what "Zhou” refers to. Lan Hua Hua was adapted from a real story. If the English readers can't understand the above information, they can't understand the plot completely. When reading the sentence "She saw a little old fellow, and retched at his home like a tomb", the interviewees can't understand the part "retched at his home like a tomb". What's more, as for "then with mutton in hand and millet cakes under her arm”, the English readers can't understand what food millet cakes actually are, let alone to see that millet cakes are a kind of precious food, usually used for treating guests of honor. That is to say, they can't see the cultural connotations of millet cakes. When their feelings are asked after they hear the English versions of northern Shaanxi folk songs, they say that there are too many words for some tunes, and the language is too formal, not suited for songs.

From the above interview, we can see sometimes our Chinese translators make no mistakes in grammar and semantics, but the English readers still can't understand the translation. Essentially the sentences are not idiomatic expression. In addition, some cultural information should be added to help the English readers to understand the translation. Finally, the language style and the poor matching of the words and the tune also influence the readers' understanding. 


\section{Main Problems in the English Versions of Northern Shaanxi Folk Songs}

According to the above interview, it can be seen that some factors prevent people from understanding the English versions thoroughly. That is to say, there exist some problems in the English versions of Northern Shaanxi folk songs.

The Inaccurate Expression. The inaccurate expression is the biggest problem in the English versions, which is mainly reflected in the following aspects: grammatical mistake, improper diction, the blending of sentence structures, the wrong logic and Chinglish. Among them improper diction and Chinglish appear more frequently. The former is either due to the wrong understanding of the source language or the improper choice in the target language. Thus some problems may come out, such as failure of transferring the original ideas, or the distraction from the original meaning, or misleading to the readers. And Chinglish is mainly due to the negative transfer of one's mother tongue. In this case, the translator deals with the target language with one's mother tongue thinking pattern. The translation produced in this way is not idiomatic. Sometimes, the English readers even can't understand the translation.

The Improper Handling of Cultural Elements. Northern Shaanxi folk songs, in the process of its production, development and spreading, absorb rich cultural elements. They usually reflect the geographical features and folk customs, which include material customs, spiritual customs and social customs. It can be said without exaggeration that Northern Shaanxi folk songs are a live picture of Northern Shaanxi folk customs. [3]Therefore, how to handle the folk culture may decide on the quality of the translation of Northern Shaanxi folk songs. Due to the cultural differences between Chinese and English, the improper handling of the cultural elements may led to the target readers' wrong understanding of the meaning, let alone the emotions and cultural background information beyond the lines.

The Lack of Colloquial Language. The English versions of Northern Shaanxi folk songs mean to be sung, so the translator should strike a balance between poems and songs. Northern Shaanxi folk songs originate from local people's daily life, and get spread from mouth to mouth. Therefore, the language is colloquial, instead of being elegant and formal, which is reflected frequently in Northern Shaanxi folk songs. Just because of the colloquial feature, there are a lot of reduplicated words and dialects. However, some English versions have a stronger flavor of poem than a song and the language is too elegant. Thus in the process of singing, those songs lose the flavor of being plain, bold and unstrained.

The Unsuitability for Singing. There are two kinds of translations of Northern Shaanxi folk songs. One is just the translation of the meaning, without the consideration of singing, which may be called the translation of the words in the song. The other not only transfers the meaning, but also takes singing into consideration, which may be called song translation and composition. The most basic difference between the two is that the latter is based on reciting, singing, and hearing because the connection between a song and a hearer is formed on the basis of hearing. [4] Therefore, the translation of Northern Shaanxi folk songs finally aims at being sung. But some translations of Northern Shaanxi folk songs only strive for the transfer of the words, failing to consider the matching of the words and the tune. That is mainly reflected in the fact that there are more syllables in the translated version, which leads to the haste of singing all the words within limited length of tune.

\section{Possible Solutions}

In view of the above problems in the English versions of Northern Shaanxi folk songs, the author proposes some suggestions as possible solutions. On one hand, the number of the English versions should be increased. Although there have existed some versions, the number is still limited. And for some famous songs, there are several versions, but for those less famous ones, there are even no English versions. That is to say, not all the Northern Shaanxi folk songs have attracted translators' attention. The distribution of the English versions is not well-balanced. On the other hand, the 
quality of the English versions needs improvement so that the English readers may better understand them. To be specific, first the translator should pay much attention to idiomatic expression. That requires the translator to learn more English idiomatic expressions, consider a lot in choosing words and sentence patterns and show a careful attitude in translation. Second, the translator should know more about Northern Shaanxi folk songs, including the cultural elements contained. When dealing with the cultural elements, the translator must take the readers' acceptability into consideration and try to make the cultural elements understood and accepted by foreigners. In the choice of the concrete method, foreignization should be given priority. If it can't do, domestication is also acceptable. Third, the language style of the versions should be colloquial and informal since the Northern Shaanxi folk songs originate from people's daily life and the local people don't use elegant and formal expression in their talk and singing. Last but not least, the end result of the English versions is for singing. When translators translate the folk songs, they should not only transfer the meaning, but also consider the matching of the words and the tunes. They should try to use similar number of syllables in the English versions so that the versions are suited for singing. If the above suggestions are accepted and really put to use, it's reasonable to believe that the English versions of Northern Shaanxi folk songs may be better understood and accepted by the English readers. And the English versions probably can go farther and become more popular abroad.

\section{References:}

[1]Wang Hongyin. Voice from the Northwest--Folk Songs of Northern Shaanxi. [M]. Beijing: Culture and Art Press, 2009: 4

[2]Han Xiaoyue, Wang Xinyu. Translation and Brief Introduction to Some Folk Songs of Northern Shaanxi. [M].Xi'an: Xi'an Press, 2013: 6

[3]Tian Ling. The Translation of the Folk Cultures in Northern Shaanxi Folk Songs.[J] Journal Of Mudanjiang University. 2010(5)

[4]Xue Fan. The Exploration and practice of Song Translation.[M]. Wu Han: Hubei Educational Press, 2002:221 